\title{
Conhecimento sobre desenvolvimento infantil em mães primíparas de diferentes centros urbanos do Brasil ${ }^{1}$
}

\author{
Maria Lucia Seidl de Moura \\ Universidade do Estado do Rio de Janeiro \\ Rodolfo de Castro Ribas Jr. \\ Universidade Federal do Rio de Janeiro \\ César Augusto Piccinini \\ Universidade Federal do Rio Grande do Sul \\ Ana Cecília de Sousa Bastos ${ }^{2}$ \\ Universidade Federal da Bahia \\ Celina Maria Colino Magalhães \\ Universidade Federal do Pará \\ Mauro Luís Vieira \\ Universidade Federal de Santa Catarina \\ Nádia Maria Ribeiro Salomão \\ Algeless Milka Pereira Meireles da Silva ${ }^{3}$ \\ Universidade Federal da Paraíba
}

Anna Karina da Silva ${ }^{4}$

Universidade Federal de Santa Catarina

\begin{abstract}
Resumo
Cognições parentais constituem importante componente do contexto sociocultural em que se dá o desenvolvimento infantil, e a literatura brasileira sobre o tema é ainda escassa. O objetivo deste estudo é analisar a relação entre conhecimento sobre desenvolvimento infantil e variáveis da mãe e do bebê. Foi estudada uma amostra de 405 mães primíparas, com filhos menores de um ano, distribuída por seis cidades em diferentes regiões do Brasil. Utilizou-se o Inventário do Conhecimento do Desenvolvimento Infantil (KIDI). Foram encontrados efeitos significativos de escolaridade materna e centro urbano. O efeito significativo de escolaridade materna foi verificado em todas as cidades, menos em Porto Alegre, possivelmente pelas políticas de atenção materno-infantil aí implementadas. Estes resultados contribuem para o conhecimento de aspectos do contexto de desenvolvimento de crianças brasileiras, e têm implicações para o planejamento de programas de intervenção que visem à promoção de saúde.
\end{abstract}

Palavras-chave: contexto sociocultural; desenvolvimento infantil; conhecimento materno; mães primíparas

\begin{abstract}
Primiparous mothers' knowledge about child development of different Brazilian urban centers. Parental cognitions are important components of the sociocultural context of child development. The Brazilian literature regarding this subject is still scarce. The purpose of this research is to analyze the relationship between knowledge of infant development and different variables concerning the mother and the infant. Four hundred and five primiparous mothers with their children less than 1 year old, in six cities of different regions of Brazil, took part in the study. The Knowledge of Infant Development Inventory (KIDI) was used. Mothers' educational level and urban center where they lived showed significant effects. This result was found for groups of all urban centers studied, with the exception of Porto Alegre. This result may reflect the different public policies concerning child and maternal care in that city. These findings contribute towards understanding important aspects of the developmental context of Brazilian children. They have implications for planning health promotion intervention programs.
\end{abstract}

Keywords: sociocultural context; child development; maternal knowledge; primiparous mothers 
$\mathrm{P}$ erspectivas contemporâneas no âmbito da psicologia do desenvolvimento têm enfatizado o papel de fatores históricos, culturais e sociais sobre o desenvolvimento humano, sem minimizar a interação destes com fatores biológicos, incluindo aqui a bagagem genética individual e da espécie (Keller, Poortinga, \& Schölmerich, 2002). Dentro dessa perspectiva, Harkness e Super têm discutido a relevância dos fatores históricos, culturais e sociais, explicitados no modelo ecológico de nichos de desenvolvimento (Harkness \& Super, 1994; Harkness \& Super, 1996; Harkness, Super, Axia, Eliaz, Palácios, \& Welles-Nyström, 2001). Esse modelo propõe que o desenvolvimento infantil processa-se em um nicho cujo eixo central é a casa (não importando a forma que esta assuma) da família (os cuidadores da criança). Esse nicho é concebido como um sistema composto por três subsistemas que se relacionam dinamicamente: o ambiente físico e social onde a criança vive (e.g., tipo de moradia, tipo de organização social da família); os costumes estabelecidos cultural e historicamente, relacionados aos cuidados e criação das crianças (e.g., a noção de infância, e do que é apropriado para crianças, as relações entre as gerações, as formas de cuidados básicos e de educar crianças); a psicologia dos que cuidam das crianças (e.g., crenças e expectativas de mães em relação a seus filhos). Para estudar as crenças ou cognições parentais, com o objetivo de entender a estrutura dos nichos de desenvolvimento e, finalmente, o próprio desenvolvimento humano, o presente estudo apóia-se nesse modelo, pressupondo-se que as crenças, como parte da psicologia dos cuidadores, afetarão as práticas de cuidado (e vice-versa), e práticas e crenças transformarão e serão transformadas pelo ambiente físico e social. É nessa dinâmica complexa que se situa o desenvolvimento infantil.

Revisões recentes da literatura sobre o tema (Ribas, 2004; Siegel \& McGillicuddy-De Lisi, 2002) têm revelado que existem controvérsias em torno da melhor maneira de definir as cognições parentais e ainda conceitos correlatos, como crenças parentais. No entanto, há certo consenso de que essas cognições atuam como mediadores, influenciando práticas de cuidado e comportamentos parentais. Esses, por sua vez, acabam tendo efeitos diversos sobre o comportamento das crianças e o desenvolvimento infantil (Ribas, Seidl de Moura, $\&$ Bornstein, 2003).

O conhecimento do desenvolvimento infantil tem um papel central no sistema de crenças parentais, e, conseqüentemente, tem influência significativa na interação entre pais e filhos. Entre outros aspectos, esse conhecimento engloba crenças acerca dos períodos mais prováveis para aquisição de habilidades motoras, perceptuais e cognitivas durante o desenvolvimento infantil; crenças acerca de que fatores podem influenciar o desenvolvimento das crianças; crenças acerca de que tipos de cuidados de higiene e segurança são importantes para a saúde das crianças. Assim, se os pais acreditam, por exemplo, que os bebês não enxergam ao nascer, é razoável supor que eles acabam por criar poucas oportunidades de estimulação visual.

Diferenças sociais e culturais nas crenças parentais acerca do desenvolvimento humano têm sido também sistematica- mente relatadas na literatura (Goodnow, 1988; Holden 1995; Miller 1988). Ninio (1979), por exemplo, verificou que mães israelenses oriundas de famílias com nível socioeconômico (NSE) mais baixo estimaram que as crianças adquirem determinadas habilidades cognitivas básicas (e.g., visão, audição, compreensão da linguagem) com uma idade significativamente maior do que a idade estimada por mães oriundas de famílias com NSE mais alto. Além disto, mães oriundas de famílias com baixo NSE avaliaram que a introdução de certos cuidados com a criança (e.g., começar a falar com o bebê, interromper a amamentação, comprar o primeiro livro) deveria ocorrer em uma idade maior do que a idade apontada por mães oriundas de famílias com alto NSE.

Ninio (1979), no estudo citado, utilizou uma composição do nível educacional e da ocupação profissional dos pais e não das mães para determinar o NSE das famílias. Essa estratégia apresenta inconvenientes. Pode-se argumentar, por exemplo, que não é possível avaliar isoladamente a importância das variáveis nível educacional e ocupação profissional para a produção dos resultados obtidos. Também não é possível entender que mecanismos relacionariam a ocupação e o nível de escolaridade dos pais com as crenças das mães. Resultados mais compreensíveis foram obtidos, por exemplo, por Benasich e Brooks-Gunn (1996), que identificaram uma correlação positiva entre nível educacional das mães e renda familiar e determinadas crenças maternas acerca do desenvolvimento infantil (e.g., idade mais provável para a aquisição de determinadas habilidades cognitivas ou comportamentais).

Bornstein, Hahn, Suwalsky e Haynes (2003) analisaram o efeito do NSE sobre comportamentos de mães e seus bebês de 5 meses, utilizando dois índices de NSE (Hollingshead Four-Factor Index of Social Status [HI] e Socioeconomic Index of_Occupations [SEI]) e componentes desses índices (educação, ocupação e renda). Os autores verificaram que o HI e o SEI apresentaram índices de correlação altos e significativos. Entretanto, uma modelagem estrutural de dados revelou que apenas o HI mostrou-se um bom preditor dos comportamentos das mães e de seus bebês. Esse estudo produziu outro resultado ainda mais revelador: o único componente do HI que se mostrou bom preditor dos comportamentos das mães e de seus bebês foi o nível de escolaridade das mães. Esses resultados ressaltam a importância da escolaridade das mães como uma variável influente sobre a cognição parental.

Em um trabalho recente, Bornstein, Hahn, Suizzo e Haynes (2003) conduziram dois estudos sobre conhecimento do desenvolvimento infantil. Ambos os estudos foram realizados com grandes amostras de mães, às quais foi administrado o Inventário de Conhecimento do Desenvolvimento Infantil (The Knowledge of Infant Development Inventory, KIDI, MacPhee, 1981). No primeiro estudo, de que participaram 1336 mães norte-americanas de crianças com 2 anos, os autores verificaram, entre outros aspectos, que o conhecimento sobre o desenvolvimento infantil podia ser significativamente associado às seguintes variáveis: idade das mães, nível educacional das mães e quantidade de leitura sobre o assunto. Não foram identificadas diferenças significativas 
entre mães de meninos e meninas, entre mães com e sem ocupação profissional remunerada, ou entre mães naturais e mães adotivas. No segundo estudo, envolvendo 2252 mães de sete países (Argentina, Bélgica, França, Israel, Itália, Japão e Estados Unidos), Bornstein e colegas verificaram diferenças no conhecimento do desenvolvimento infantil entre diversas amostras. A leitura de material escrito, a participação em turmas para gestantes, e o apoio de companheiros, amigos e vizinhos também contribuíram significativamente para o conhecimento do desenvolvimento infantil. Entre outros aspectos, esses resultados ressaltam a influência da escolaridade das mães, sugerem a influência de variáveis culturais (inferidas pelas diferenças transnacionais) e apontam para a influência de outras variáveis, como a participação em cursos de gestantes.

Apoiando a idéia de diferenças culturais no conhecimento sobre o bebê, o estudo de Goodnow, Cashmore, Cotton e Knight (1984) indicou que mães de origem australiana e libanesa, residentes na Austrália, apresentaram diferenças nas avaliações acerca dos marcos do desenvolvimento - idades em que se espera a aquisição de determinadas habilidades. Os resultados obtidos nesse estudo devem, entretanto, ser avaliados com reservas, já que as mães de origem libanesa possuíam nível de escolaridade mais baixo que as mães de origem australiana. Assim, não é possível concluir se essas diferenças estavam relacionadas à origem étnica, ao nível educacional ou a uma combinação dessas variáveis. Certamente essas variáveis são relacionadas e, junto com outras, vão compor o contexto sociocultural do desenvolvimento, mas há que tentar estudar seus efeitos de forma separada e a possível interação entre esses efeitos, para evitar conclusões equivocadas. Isso foi amplamente verificado nas pesquisas da década de 1970, revistas por Rogoff e Chavajay (1995). Diferenças em avaliações cognitivas atribuídas à origem cultural em geral, eram, na verdade, resultantes de diferenças no acesso à escolaridade.

Utilizando um delineamento de pesquisa mais complexo, Harwood, Schoelmerich, Ventura-Cook, Schulze e Wilson (1996) investigaram simultaneamente a influência do status étnico (anglo-saxão ou latino-americano) e do NSE sobre as crenças de mães acerca de metas desejáveis para o processo de socialização e para o comportamento infantil. Tanto o status étnico quanto o NSE mostraram ter influência significativa sobre as metas de socialização das mães. Esses autores, entretanto, investigaram apenas a influência do NSE (calculado a partir do nível educacional e a ocupação profissional das mães e seus parceiros) em sua investigação, não tendo avaliado a importância específica do nível educacional das mães para a produção dos resultados relatados.

Ribas et al. (2003) realizaram uma revisão de literatura sobre o conhecimento parental e identificaram algumas tendências principais: 1) Os achados endossam a relação entre o nível socioeconômico e o conhecimento parental. Dentre os diversos indicadores de NSE, o nível educacional dos pais, em especial o da mãe, é o que tem maior valor discriminante em estudos sobre desenvolvimento infantil. 2) O conhecimento de pais sobre desenvolvimento infantil afeta os com- portamentos parentais e, conseqüentemente, o desenvolvimento da criança. Pais que têm acesso a conhecimento sobre o desenvolvimento infantil, e suas principais etapas, têm expectativas mais razoáveis sobre o comportamento de seus filhos e, com isso, têm maior probabilidade de comportar-se de forma mais adequada (e.g., não pensar que é possível o controle de esfíncteres com poucos meses de idade e, portanto, não esperar que um bebê possa ser treinado a usar o banheiro nesse período do desenvolvimento). Os pais podem também planejar melhor as situações que propiciem seu desenvolvimento e tomar decisões mais apropriadas sobre os cuidados infantis. Em contraste, pais que desconhecem essas etapas ou sequiências esperadas sofrem mais estresse pela divergência entre suas expectativas e as possibilidades reais das crianças, e tendem a tomar decisões pouco adequadas, que podem até ter sérias conseqüências para a criança. 3) O conhecimento parental sobre o desenvolvimento infantil parece ter relação com o desenvolvimento infantil nos primeiros anos.

O estudo de Benasich e Brooks-Gunn (1996) contempla os três tipos de relações mencionados acima, fornecendo evidências que relacionam conhecimentos parentais, comportamentos parentais e desenvolvimento infantil. Esses autores identificaram, em uma amostra de mães, relações significativas entre conhecimentos e atitudes e o nível educacional das mães; entre conhecimentos e atitudes parentais e comportamentos parentais; e entre conhecimentos e atitudes parentais e o desenvolvimento infantil.

A revisão de Ribas et al. (2003) revelou ainda que, apesar de ter havido um crescimento da literatura sobre crenças e práticas parentais, essas pesquisas têm se restringido aos Estados Unidos, com poucas exceções em outros países. A literatura brasileira também é ainda pouco expressiva sobre este tema. De fato, crenças de adultos brasileiros sobre desenvolvimento infantil têm sido investigadas por diversos pesquisadores (e.g., Bastos, 2001; Batista, 1984; BiasoliAlves, 1997; Gomes, Soares, Ribas, \& Seidl de Moura, 1998; Nogueira, 1988; Preuss, 1986; Ribas \& Seidl de Moura, 1995; Seidl de Moura, Ribas \& Ribas, 1998; Trindade, 1993). Entretanto, poucos desses trabalhos trataram especificamente do conhecimento materno sobre desenvolvimento infantil, e nenhum deles adotou uma perspectiva comparativa intercultural.

Ribas et al. (2003) utilizaram uma versão do Inventário do Conhecimento do Desenvolvimento Infantil (KIDI) de MacPhee (1981). Participaram desse estudo 64 mães primíparas do Rio de Janeiro, membros de famílias intactas, maiores de 18 anos, com filhos de 5 meses $\mathrm{O}$ escore médio obtido pelas mães da amostra brasileira no $\operatorname{KIDI}(M=0,63, D P=0,11)$ foi um pouco mais baixo do que o escore médio obtido no estudo original de MacPhee (1981) com 226 mães norte-americanas $(M=0,72, D P=0,12)$. Contudo, essa diferença deve ser avaliada com reservas, já que, em função das medidas utilizadas, não foi possível comparar o nível de escolaridade e o NSE das amostras brasileira e amostra norte-americana. Corroborando estudos anteriores, os autores verificaram ainda que o nível educacional materno foi o melhor preditor dos escores do KIDI. Apesar de interessantes, esses resultados devem também ser considerados com cautela, levando-se em 
consideração a especificidade (idade dos filhos) e o tamanho da amostra envolvida. Assim, pouco se sabe a respeito da influência da idade dos bebês, de eventuais variações regionais e de outras variáveis que possam ser associadas ao conhecimento sobre o desenvolvimento infantil.

Campos e Gomes (1996), por exemplo, apontaram que diferenças sociais extremas existem e sempre existiram no Brasil entre as realidades regionais, populações urbanas e rurais, e entre classes sociais. As autoras consideram que "é possível identificar [...] um movimento que está levando a certa homogeneidade - e assim a hegemonia do sistema simbólico urbano e 'moderno' tende a encobrir as diferenças entre as condições objetivas de vida" (p. 143). Na área de cognições parentais, no entanto, sem estudos mais amplos, com amostras que cubram pelo menos em parte as diversidades regionais do Brasil, não se pode concluir, nem na direção da homogeneidade, nem da diversidade ou de diferenças relativas a grupos culturais ou de nível socioeconômico diversos.

A partir de uma perspectiva sociocultural, do modelo de nicho de desenvolvimento e da análise das evidências da literatura que foram discutidas, considera-se que vários aspectos justificam a condução do presente estudo. As crenças maternas são um importante aspecto do nicho de desenvolvimento. Dentro de um sistema de crenças maternas, o conhecimento sobre desenvolvimento infantil tem um papel relevante e pode afetar práticas e, dessa forma, o desenvolvimento de crianças. A literatura brasileira ainda não é extensa e não há estudos específicos sobre o tema. Buscou-se obter informações sobre conhecimento do desenvolvimento infantil, a partir de uma amostra ampla de mães brasileiras, residentes em diferentes centros urbanos, escolha feita porque se considera que é no contexto urbano que vive a maioria da população brasileira. A participação de mães residentes em diferentes centros urbanos permite avaliar diferenças regionais, questão raramente investigada em pesquisas psicológicas brasileiras. Finalmente, o estudo foi delineado de forma a contribuir para um melhor entendimento acerca da influência específica de determinadas variáveis sobre o conhecimento do desenvolvimento infantil, levando em consideração especificidades regionais.

Buscou-se atingir os seguintes objetivos: a) avaliar o conhecimento sobre o desenvolvimento infantil de mães brasileiras primíparas com crianças de 0 a 1 ano de idade, b) examinar as relações entre o nível socioeconômico das famílias, o nível educacional das mães e o conhecimento sobre o desenvolvimento infantil de mães, c) investigar possíveis diferenças regionais no conhecimento sobre o desenvolvimento infantil, e d) investigar a influência das variáveis idade da mãe, sexo do bebê e idade do bebê sobre conhecimento sobre o desenvolvimento infantil de mães em diversas regiões.

\section{Método}

\section{Amostra}

Participaram do estudo 405 mães primíparas, residentes em seis centros urbanos brasileiros (Belém, Itajaí, João Pessoa, Porto Alegre, Rio de Janeiro e Salvador), com idades entre 18 e 47 anos $(M=26,30, D P=5,77)$. As mães possuíam diferentes níveis educacionais e socioeconômicos, e um filho ou uma filha com até 1 ano de idade. A Tabela 1 apresenta o número de mães que participaram da pesquisa em cada centro urbano, e dados sociodemográficos da amostra, tais como: idade e sexo dos bebês, idade, nível educacional e prestígio ocupacional das mães, e nível socioeconômico das famílias. Dentre as mães que participaram da pesquisa, 233 (57,5\% das mães) possuíam alguma ocupação profissional. As mães que possuíam ocupação profissional exerciam funções com prestígio ocupacional compatível com sua escolaridade (ver correlações indicadas na Tabela 2). Dessa forma, o prestígio ocupacional das mães oscilou dentro de um contínuo, que foi de ocupações de baixo prestígio (faxineiras, empregadas domésticas, balconistas) até ocupações de alto prestígio (e.g., economistas, médicas, advogadas).

Tabela 1

Descrição da amostra

\begin{tabular}{|c|c|c|c|c|c|c|c|c|}
\hline & Belém & Itajaí & João Pessoa & Porto Alegre & Rio de Janeiro & Salvador & Total & $\mathrm{F}$ e $\chi^{2}$ \\
\hline $\mathrm{N}$ & 60 & 109 & 62 & 62 & 59 & 53 & 405 & \\
\hline Idade da mãe (anos) & $24,72(4,22)$ & $25,91(5,94)$ & $24,34(4,23)$ & $29,29(6,61)$ & $28,58(6,09)$ & $25,12(5,04)$ & $26,30(5,77)$ & $8,82^{*}$ \\
\hline Escolaridade da mãe ${ }^{1}$ & $4,32(1,58)$ & $4,20(1,55)$ & $4,56(1,28)$ & $4,97(1,37)$ & $5,19(1,34)$ & $3,61(2,07)$ & $4,46(1,60)$ & $7,88^{*}$ \\
\hline Ocupação da mãe ${ }^{2}$ & $6,40(2,79)$ & $6,71(1,85)$ & $5,53(2,52)$ & $6,20(2,10)$ & $6,87(1,66)$ & $4,36(3,32)$ & $6,21(2,36)$ & $4,66^{*}$ \\
\hline Idade do bebê (meses) & $7,15(3,34)$ & $6,54(3,77)$ & $5,68(3,71)$ & $6,73(3,43)$ & $7,27(3,65)$ & $6,00(3,10)$ & $6,57(3,57)$ & 1,84 \\
\hline Porcentagem de meninas** & 40,68 & 53,21 & 40,32 & 42,55 & 52,54 & 49,06 & 47,30 & 4,92 \\
\hline Status socioeconômico ${ }^{3}$ & $\begin{array}{c}43,04 \\
(16,18)\end{array}$ & $\begin{array}{c}41,14 \\
(13,56)\end{array}$ & $\begin{array}{c}39,15 \\
(14,03)\end{array}$ & $46,43(13,43)$ & $47,04(13,47)$ & $\begin{array}{c}33,15 \\
(19,61)\end{array}$ & $\begin{array}{c}42,21 \\
(15,00)\end{array}$ & $5,09^{*}$ \\
\hline
\end{tabular}

\footnotetext{
${ }^{1}$ Escala de 7 níveis adaptada de Hollingshead (1975)

${ }^{2}$ Escala de 9 níveis adaptada de Hollingshead (1975)

${ }^{3}$ Hollingshead's Four-Factor Index of Social Status

$* \mathrm{~F}: \mathrm{p}<0,01$

$* * \chi^{2}$
} 
Tabela 2

Correlações entre variáveis sociodemográficas maternas, idade do bebê e escore no KIDI

\begin{tabular}{|c|c|c|c|c|c|}
\hline & 1 & 2 & 3 & 4 & 5 \\
\hline \multicolumn{6}{|l|}{ 1. Nível Socioeconômico } \\
\hline 2. Escolaridade da Mãe ${ }^{2}$ & $0,75^{*}$ & & & & \\
\hline 3. Ocupação da Mãe ${ }^{3}$ & $0,89 *$ & $0,68^{*}$ & & & \\
\hline 4. Idade da Mãe (anos) & $0,35^{*}$ & $0,46^{*}$ & $0,39^{*}$ & & \\
\hline 5. Idade do Bebê (meses) & 0,07 & 0,07 & $-0,05$ & $0,16^{*}$ & \\
\hline 6. $\mathrm{KIDI}^{4}$ & $0,35^{*}$ & $0,40^{*}$ & 0,12 & $0,16^{*}$ & $0,15^{*}$ \\
\hline
\end{tabular}

${ }^{1}$ Hollingshead's Four-Factor Index of Social Status

${ }^{2}$ Escala de 7 níveis adaptada de Hollingshead (1975)

${ }^{3}$ Escala de 9 níveis adaptada de Hollingshead (1975)

${ }^{4}$ Proporção de respostas corretas

$* \mathrm{p}<0,05$

\section{Instrumentos}

Nível educacional, prestígio ocupacional e nível socioeconômico. Foi utilizado um questionário padronizado para coletar informações sociodemográficas das famílias (e.g., idade, nível educacional e profissão da mãe e seu companheiro, idade e sexo dos filhos). As escalas adaptadas de Hollingshead (1975) foram utilizadas para avaliar o nível educacional, prestígio ocupacional e o nível socioeconômico da amostra. A validade dessas escalas para utilização no Brasil já foi estabelecida em estudos anteriores (ver, por exemplo, Ribas et al., 2003; Ribas, Seidl de Moura, Gomes, Soares, \& Bornstein 2003; Ribas et al., 2000b).

O nível educacional levou em conta o número de anos de escolarização formal e foi inicialmente codificado em uma escala de 7 níveis (1, primeiro grau incompleto; a 7, pósgraduação). O prestígio ocupacional foi avaliado com base em uma lista com aproximadamente 450 atividades profissionais existentes no Brasil, e foi tabulada em uma escala de 9 níveis. Empregados não-qualificados e empregadas domésticas, por exemplo, receberam a menor pontuação da escala, 1; ao passo que executivos e profissionais liberais receberam a maior pontuação da escala, 9 .

A partir da pontuação na escala, utilizou-se o Hollingshead Four Factor Index of Socioeconomic Status (HI, Índice Quadrifatorial de Status Socioeconômico, Hollingshead, 1975), um dos índices de nível socioeconômico mais utilizados em pesquisas em psicologia do desenvolvimento. O HI é calculado, para cada indivíduo, somando-se o nível educacional multiplicado por 3 com o nível de prestígio ocupacional multiplicado por 5. Dessa forma o HI produz um escore compósito que pode variar de 8 a 66. Para famílias nucleares, com apenas um cônjuge (pai ou mãe) empregado, o HI é calculado com base no nível educacional e prestígio ocupacional do cônjuge empregado. No caso de ambos os cônjuges serem empregados, são computados escores individuais para cada um dos cônjuges e é calculada uma média simples destes escores.
Conhecimento sobre o desenvolvimento Infantil. A versão em português do Inventário do Conhecimento do Desenvolvimento Infantil (KIDI, Knowledge of Infant Development Inventory, MacPhee, 1981), traduzida e adaptada por Ribas, Seidl de Moura, Gomes e Soares (2000a), foi utilizada. Esse instrumento permite a avaliação de uma ampla gama de conhecimentos relacionados a crianças de até 2 anos que incluem: períodos mais prováveis para aquisição de habilidades motoras, perceptuais e cognitivas; fatores relacionados ao desenvolvimento; práticas parentais; cuidados de alimentação, higiene e segurança.

O inventário possui 75 questões, 48 das quais solicitam que o respondente escolha uma entre três respostas (concordo, discordo, ou não estou certo/a) sobre umas séries de declarações (e.g., "Em geral, os bebês não podem ver e ouvir ao nascerem"). Outras 20 questões pedem que o respondente escolha uma entre quatro respostas (concordo, mais jovem, mais velho, não tenho certeza) relacionadas a afirmações sobre quando uma criança deveria adquirir uma determinada competência (e.g., "As crianças normalmente já estão andando por volta dos 12 meses de idade"). Finalmente, 7 questões solicitam que o respondente escolha uma entre cinco possíveis respostas (e.g., "A melhor maneira de baixar a febre de um bebê é:" "a. Colocar um pano frio na testa do bebê", "b. Colocar mais roupas no bebê.”, "c. Dar gotas de remédio contra a febre.", “d. Dar muita vitamina C para o bebê.", “e. Não tenho certeza"). O KIDI foi originalmente padronizado em quatro grupos: estudantes universitários, mães, psicólogos e pediatras norte-americanos (MacPhee, 1981). Em uma amostra de mães foi observada boa fidedignidade para proporção de respostas corretas (Alfa $=0,82 ;$ Guttman Split-half $=0,85)$. Ribas et al. (2000a) relataram resultados semelhantes para a versão adaptada do KIDI (Alfa $=0,81$; Guttman Splithalf $=0,80$ ) obtidos em uma amostra de 186 adultos, homens e mulheres, com e sem filhos. No presente estudo, envolvendo 405 mães, foi observado um índice alfa de 0,70 e um Guttman Split-half de 0,63.

\section{Coleta de Dados}

A amostra foi obtida a partir de indicações pessoais e de pediatras, e em instituições (por exemplo, Unidade de Amamentação do Hospital Universitário Betina de Ferro, Belém; Centro de Referência à Saúde da Criança e da Mulher, Itajaí; Posto de Saúde situado em bairro popular, Salvador). Os instrumentos foram aplicados individualmente, por pesquisadores e assistentes de pesquisa (alunos de graduação e pós-graduação, devidamente treinados para essa tarefa), na casa das mães (quando tinham sido indicadas) ou nas instituições onde as mães foram contatadas. Em cada aplicação, as mães foram informadas sobre os objetivos da pesquisa, sobre o sigilo e confidencialidade das suas respostas e sobre o caráter voluntário da participação e preencheram formulários de consentimento informado. O presente estudo é parte de um projeto mais amplo que teve aprovação do Comitê Nacional de Ética em Pesquisa - CONEP (Seidl de Moura, 1999).

A coleta de dados foi conduzida nos diferentes centros urbanos de forma a obter informações de mães com diferen- 
tes níveis educacionais e econômicos, bebês até 1 ano de idade (distribuídos em três subgrupos de idade) e o número de meninos e meninas (ver Tabela 1). Ao final da coleta de dados as amostras dos diferentes centros foram analisadas e se verificou que se mostravam equivalentes em relação à idade dos bebês, $F_{(5,397)}=1,84$ (ns), e à proporção de meninos e meninas, $\chi_{(5,389)}^{2}=4,92(\mathrm{~ns})$. Diferiam significativamente, entretanto, no que se refere à idade, à escolaridade e ao prestígio ocupacional das mães, bem como ao nível socioeconômico das famílias (ver Tabela 1).

\section{Resultados e Discussão}

\section{Análises preliminares}

Antes da realização das análises principais, foram conduzidas análises de todas as variáveis envolvidas no estudo com o objetivo de avaliar as distribuições (normalidade) e identificar valores extremos (outliers). Os dados de uma única mãe foram descartados porque se verificou um resultado no KIDI (proporção de respostas certas) extremo (mais de três desvios padrão abaixo da média do grupo). As médias (com desvio padrão entre parênteses) dos escores no KIDI obtidos pelos diferentes centros urbanos e amostra total foram as seguintes: Belém, 0,61 (0,09), Itajaí, 0,58 (0,07), João Pessoa, 0,65 (0,08), Porto Alegre, 0,69, (0,06), Rio de Janeiro, 0,66 (0,08), Salvador, 0,67 (0,08); total da amostra, 0,64 (0,09).

As variáveis nível socioeconômico das famílias (NSE), idade das mães, escolaridade das mães e prestígio ocupacional das mães poderiam ser utilizadas na análise principal como fatores em uma ANCOVA em que a proporção de respostas corretas no KIDI seria variável dependente. Entretanto, dessas variáveis, decidiu-se incluir apenas a escolaridade das mães como um fator na análise principal.

Três justificativas orientaram essa decisão. Em primeiro lugar, tal como previsto, foram identificadas correlações altas e significativas entre essas quatro variáveis (ver Tabela 2). Esses resultados são similares aos observados por Bornstein et al. (2003) e por Ribas et al. (2003). A inclusão dessas variáveis em um mesmo modelo acarretaria, portanto, um problema de multicolinearidade. Em segundo lugar foi verificado ainda que o nível de educação das mães apresentou maior índice de correlação com o conhecimento do desenvolvimento infantil $(R=0,40)$ do que o NSE $(R=0,35)$ e a idade das mães $(R=$ $0,16)$, e que o prestígio ocupacional das mães não se correlacionou com o conhecimento do desenvolvimento infantil (ver Tabela 2). Em terceiro lugar, por definição, NSE é um compósito, calculado utilizando a escolaridade e ocupação de pais e mães, e a escolaridade da mãe oferece um indicador desse compósito (Bornstein et al., 2003). Finalmente, no presente estudo observou-se que a proporção de falta de dados acerca da educação da mãe $(1,20 \%)$ foi muito inferior à proporção de falta de dados acerca do NSE das famílias $(13,80 \%)$. Em função disso, a inclusão do NSE como fator reduziria significativamente o tamanho da amostra para a análise.

A escolaridade das mães foi recodificada em uma escala de três níveis para ser introduzida na $\operatorname{ANCOVA}(1$, primeiro grau incompleto ou completo; 2 , segundo grau incompleto ou completo; 3 , curso superior incompleto ou completo $e$ pós-graduação).

Considerando que a idade do bebê era uma candidata a covariável na análise principal e, tendo em vista a correlação significativa entre esta variável e o KIDI (ver Tabela 2), decidiu-se por incluir a idade dos bebês na análise principal como covariável.

\section{Análise principal}

Foi conduzida uma análise de covariância (ANCOVA). A idade dos bebês foi introduzida no modelo como covariável, a escolaridade das mães, o local de residência das mães (Centro Urbano, CEU), bem como o sexo dos bebês foram introduzidos no modelo como fatores preditores. A variável dependente foi o número de acertos no KIDI. A Tabela 3 apresenta os resultados da ANCOVA. A análise confirmou o efeito significativo da covariável idade dos bebês e revelou efeitos significativos dos fatores escolaridade da mãe e centro urbano. Tanto a escolaridade materna quanto o CEU produziram efeitos significativos sobre o KIDI. O sexo do bebê não apresentou efeito significativo sobre o KIDI. Foi identificado, ainda, o efeito significativo da interação entre escolaridade da mãe e centro urbano, indicando que o efeito da escolaridade sobre o KIDI variou de acordo com o centro urbano onde a mãe reside.

Tabela 3

Análise de covariância para conhecimento sobre o desenvolvimento infantil (KIDI)

\begin{tabular}{lrc}
\hline \multicolumn{1}{c}{ Fonte } & $g l$ & $F$ \\
\hline Modelo Corrigido & 35 & $6,84^{*}$ \\
Idade do Bebê (IB) & 1 & $8,66^{*}$ \\
Centro Urbano (CEU) & 5 & $16,60^{*}$ \\
Escolaridade da Mãe (EM) & 2 & $34,54^{*}$ \\
Sexo do Bebê (SB) & 1 & 0,23 \\
CEU X EM & 10 & $2,26^{*}$ \\
CEU X SB & 5 & 0,43 \\
EM X SB & 2 & 2,67 \\
CEU X EM X SB & 9 & 1,06 \\
Erro & 345 & \\
Total Corrigido & 380 & \\
\hline
\end{tabular}

$$
\begin{aligned}
& { }^{1} \mathrm{R}^{2}=0,41 \quad \mathrm{R}^{2} \text { (ajustado) }=0,35 \\
& * \mathrm{p}<0,05
\end{aligned}
$$

Fica confirmada a importância da escolaridade materna, que parece ser uma variável relevante no nicho de desenvolvimento infantil, e que se mostra correlacionada às cognições parentais, e aos conhecimentos sobre desenvolvimento infantil. A idade do bebê, mesmo nessa faixa restrita do primeiro ano de vida, parece também afetar as cognições maternas, talvez sensibilizando as mães de forma diferencial. Assim, como era esperado, quanto mais velho o bebê, maiores os conhecimentos adquiridos pela mãe. Isso sugere que não é só a mãe que influencia o bebê, mas que pistas do bebê ou características dele também tendem a afetar processos da mãe. 
Dentre essas características, a idade parece ser mais importante que o próprio sexo do bebê, pelo menos quando se trata de conhecimento sobre seu desenvolvimento. Outro resultado relevante, no entanto, é a verificação de um efeito significativo do local de residência da mãe, ou Centro Urbano, sobre seu conhecimento sobre desenvolvimento infantil. Isto sugere que existem diferenças sociais e culturais em relação a essa variável. Considerando-se esse resultado junto com o do efeito da escolaridade materna (um dos aspectos indicadores de nível socioeconômico) os dados apóiam a idéia de que pode haver mais diversidade do que homogeneidade entre as mães brasileiras, pelo menos no que ser refere à variável estudada.

Visando esclarecer o efeito do centro urbano sobre o conhecimento das mães, foi conduzida uma ANCOVA sobre os dados de cada centro urbano. A idade dos bebês foi mantida no modelo como covariável, e a escolaridade das mães e o sexo dos bebês foram introduzidos como fatores preditivos/ou como variáveis independentes. As análises produziram resultados bastante semelhantes em cinco centros urbanos (Belém, Itajaí, João Pessoa, Rio de Janeiro e Salvador). Nesses centros urbanos verificou-se efeito significativo $(p<0,05)$ da covariável idade dos bebês $(F s=2,88$, $3,87,5,09,3,71,7,70$, respectivamente) e do fator escolaridade das mães ( $F s=5,25,8,56,11,70,8,21,17,92$, respectivamente). Não foram detectados efeitos de interações. Nos resultados da análise realizada com os dados obtidos em Porto Alegre não se verificou efeito significativo da covariável idade dos bebês, nem do fator escolaridade das mães. Também não foram detectados efeitos de interações nessa amostra.

Assim, com exceção da amostra de Porto Alegre, nas demais se encontrou resultados que acompanham a tendência indicada na literatura, em relação à escolaridade materna e sua relação com as cognições maternas. $\mathrm{O}$ fato disso não ter sido verificado na amostra de Porto Alegre é intrigante e indica que outros fatores podem estar intermediando a relação entre escolaridade materna e conhecimentos sobre o bebê. Na verdade, no estudo de Bornstein et al. (2003), que examinou diferenças entre amostras de vários paises, foi encontrado que diversos fatores além da escolaridade materna (e.g., leitura de material escrito, participação em grupos de gestantes e apoio dos companheiros, amigos e vizinhos), também contribuíram significativamente para o conhecimento do desenvolvimento infantil, apontando para a influência de variáveis culturais nas cognições maternas. De qualquer modo os achados de Porto Alegre suscitaram discussões e podem ter interessantes implicações para o planejamento de ações promotoras de saúde a partir de estudos sobre cognições maternas. Porto Alegre é a segunda capital em qualidade de vida do país, se considerarmos o Índice de Desenvolvimento $\mathrm{Hu}-$ mano (IDH), e apresenta o maior índice entre os centros urbanos investigados no presente estudo 5 . Dentro deste quadro, é possível que a política de atendimento materno-infantil da cidade, traduzida em programas da Prefeitura, pode estar propiciando às novas mães conhecimentos específicos sobre o seu bebê. Isto estaria ocorrendo com todas as mães independentes do seu NSE e escolaridade. Se por um lado, comumente só as de nível mais alto tem acesso a médicos, psicólogos, revistas e outras informações privilegiadas, em Porto Alegre estas informações podem estar sendo mais socializadas. Dentro deste quadro, é possível que a política de atendimento infantil da cidade, traduzida em programas da Prefeitura, propicie conhecimentos às novas mães sobre o seu bebê. Isto estaria ocorrendo com todas as mães, independentemente do NSE das famílias e da escolaridade das mães. Como parte de uma região geográfica extensamente povoada por diversos grupos de imigrantes europeus, é possível que isto possa contribuir para explicar, pelo menos parcialmente, as diferenças encontradas a respeito dos conhecimentos maternos.

\section{Conclusões}

Este estudo constituiu uma iniciativa de certo modo pioneira no Brasil, pelo menos na área de psicologia do desenvolvimento. Nasceu da convicção dos autores da necessidade de se investigar interculturalmente aspectos e processos do desenvolvimento de diversos grupos no país (além, naturalmente, de realizar comparações com amostras de outros países). Foi possível reunir pesquisadores e estudar uma amostra que incluiu participantes de quase todas as regiões do Brasil, à exceção da região Centro-Oeste. Como experiência inicial de colaboração, o foco foi apenas um aspecto do nicho do desenvolvimento inicial: cognições de mães que estão tendo seu primeiro filho, ainda em seu primeiro ano. Dentre essas cognições, o conhecimento sobre desenvolvimento infantil foi investigado, buscando-se relacioná-lo com variáveis da mãe e do próprio bebê. Novos estudos abordarão outros processos e variáveis. Espera-se com isso ampliar a compreensão do contexto sociocultural do desenvolvimento inicial de crianças brasileiras.

Os resultados permitem algumas conclusões. Em primeiro lugar, o conjunto das análises realizadas demonstra, mais uma vez, a importância da escolaridade materna, variável comprovadamente associada ao conhecimento que essas mães possuem sobre desenvolvimento infantil. Esse conhecimento, conforme a literatura, tem impacto importante sobre os processos de desenvolvimento infantil, tornando-se fundamental, por conseguinte, priorizar e ampliar as oportunidades de educação para mulheres e mães. Caso se confirmem, em estudos posteriores, as hipóteses levantadas nesta investigação, os resultados de Porto Alegre podem indicar que a oferta continuada de uma assistência adequada pode favorecer a qualidade das interações mães-bebês, fazendo com que se revertam tendências prevalecentes em outras regiões. Ao se desenvolver e implementar políticas públicas de atendimento materno-infantil eficazes, é possível que se consiga diminuir até certo ponto o papel negativo da baixa escolaridade sobre o conhecimento infantil, fornecendo às mães oportunidades de ampliar seu conhecimento sobre desenvolvimento infantil.

Por fim, as diferenças reveladas no presente estudo sobre os centros urbanos investigados confirmam o que foi previsto: que os grupos de mães das diferentes regiões urbanas brasileiras não são homogêneos. Reafirma-se, portanto, 
o quão oportuno se faz conduzir estudos interculturais como o que ora se descreve. Variações sociais e culturais importantes podem ser hipotetizadas e devem ser consideradas pelos que se dedicam a estudar desenvolvimento infantil e aqueles que desejam desenvolver e implementar programas de promoção de saúde em nosso país.

\section{Referências}

Bastos, A. C. S. (2001). Modos de partilhar: a criança e o cotidiano da família. Taubaté: Cabral Editora Universitária.

Batista, S. M. (1984). Maternidade e exercício profissional. Arquivos Brasileiros de Psicologia, 36, 45-58.

Benasich, A. A., \& Brooks-Gunn, J. (1996). Maternal attitudes and knowledge of child-rearing: associations with family and child outcomes. Child Development, 67, 1186-1205.

Biasoli-Alves, Z. M. M. (1997). Famílias brasileiras do Século XX: os valores e as práticas da educação da criança. Temas em Psicologia, 3, 33-40.

Bornstein, M. H., Hahn, C. -S., Suwalsky, J. T. D., \& Haynes, O. M. (2003). The Hollingshead four-factor index of social status and the socioeconomic index of occupations. In M. H. Bornstein \& R. H. Bradley (Orgs.), Socioeconomic status, parenting, and child_development (pp. 29-81). Mahwah, New Jersey: Lawrence Erlbaum.

Bornstein, M. H., Hahn, C. -H., Suizzo, M., Haynes, M. (2003). Mothers' knowledge about child development and childrearing: national and crossnational studies. Manuscrito não-publicado, National Institute of Child Health and Human Development.

Campos, M. M., \& Gomes, J. V. (1996). Brazilian children: images, conceptions, projects. In C. P. Hwang, M. E. Lamb, \& I. E. Sigel (Orgs.), Images of childhood (pp. 143-166). Mahwah, New Jersey: Lawrence Erlbaum.

Gomes, A. P. do N., Soares, I. D., Ribas Jr., R. C., \& Seidl de Moura, M. L. (1998) Concepções acerca das competências do bebê recém-nascido: a influência de gênero, paternidade e formação profissional [Resumo]. In Sociedade Brasileira de Psicologia (Org.), Resumos de Comunicações Científicas da XXVIII Reunião Anual de Psicologia (p. 129). Ribeirão Preto: Autor.

Goodnow, J. J. (1988). Parents' ideas, actions, and feelings: models and methods from developmental and social psychology. Child Development, 59, 286-320.

Goodnow, J. J., Cashmore, J., Cotton, S., \& Knight, R. (1984). Mothers' developmental timetables in two cultural groups. International Journal of Psychology, 19, 193-205.

Harkness, S., \& Super, C. M. (1994). Developmental niche: a theoretical framework for analyzing the household production of health. Social Science and Medicine, $38,219-226$

Harkness, S., \& Super, C. M. (1996). (Orgs.), Parents'cultural belief systems: their origins, expressions, and consequences. Nova York: Guilford.

Harkness, S., Supper, C., Axia, V., Eliaz, A., Palácios, J., \& Welles-Nyström, B. (2001). Cultural pathways to successful parenting. International Society for the Study of Behavior Development Newsletter, 1(38), 9- 13.

Harwood, R. L., Schoelmerich, A., Ventura-Cook, E., Schulze, P. A., \& Wilson, A. (1996). Culture and class influences on Anglo and Puerto Rican mothers' beliefs regarding long-term socialization goals and child behavior. Child Development, 67, 2446-2461.

Holden, G. W. (1995). Parental attitudes toward childrearing. In M. H. Bornstein (Org.), Handbook of parenting, Vol. 3: Status and social conditions of parenting (pp. 359-377). Nova York: Lawrence Erlbaum.
Hollingshead, A. B. (1975). The four-factor index of social status. Manuscrito não-publicado, Yale University.

Keller, H., Poortinga, Y. H., \& Schölmerich, A. (2002). (Orgs.). Between culture and biology. Cambridge: Cambridge University Press.

MacPhee, D. (1981). Manual for the Knowledge of Infant Development Inventory. Manuscrito não-publicado, University of North Carolina.

Miller, S. A. (1988). Parents' beliefs about children's cognitive development. Child Development, 59, 259-281.

Ninio, A. (1979). The naive theory of the infant and other maternal attitudes in two groups in Israel. Child Development, 50, 976-980.

Nogueira, Y. (1988). Atitudes maternas: estudo do PARI (Parental Attitude Research Instrument) em amostra brasileira. Arquivos Brasileiros de Psicologia, 40, 48-62.

Preuss, M. R. (1986). Atitudes maternas e tipo de cuidado alternativo escolhido por mães que trabalham fora. Psicologia: Teoria Pesquisa, 2, 213-225.

Ribas, A F. P., \& Seidl de Moura, M. L. (1995). Construção de um questionário para o estudo da concepção de adultos acerca das competências do recémnascido. Arquivos Brasileiros de Psicologia, 47(2), 89-99.

Ribas, R. C., Jr. (2004). Cognições de mães brasileiras acerca da maternidade, da paternidade e do desenvolvimento humano: uma contribuição ao estudo da Psicologia parental. Tese de Doutorado não-publicada, Universidade do Estado do Rio de Janeiro.

Ribas, R. C., Jr., Seidl de Moura, M. L., \& Bornstein, M. H. (2003). Socioeconomic status in Brazilian psychological research. Part 2: SES and parenting knowledge. Estudos de Psicologia, 8, 385-392.

Ribas, R. C., Jr., Seidl de Moura, M. L., Gomes, A. A. N., \& Soares, I. D. (2000a). Adaptação brasileira do Inventário de Conhecimento sobre o Desenvolvimento Infantil de David Macphee [Resumo]. In Sociedade Brasileira de Psicologia do Desenvolvimento (Org.), Anais - III Congresso Brasileiro de Psicologia do Desenvolvimento (p. 183). Niterói, RJ: Autor.

Ribas, R. C., Jr., Seidl de Moura, M. L., Gomes, A. A. N., Soares, I. D., \& Bornstein, M. H. (2003). Socioeconomic status in Brazilian psychological research. Part 1: validity, measurement, and application. Estudos de Psicologia, 8, 375-383.

Ribas, R. C., Jr., Seidl de Moura, M. L., Gomes, A. A. N., Soares, I. D., Pontes, P. C., \& Silveira, R. L. (2000b). Avaliação do status socioeconômico na pesquisa psicológica brasileira: tendências e recomendações [Resumo]. In Sociedade Brasileira de Psicologia do Desenvolvimento (Org.), Anais - III Congresso Brasileiro de Psicologia do Desenvolvimento (p. 183). Niterói, RJ: Autor.

Rogoff, B., \& Chavajay, P. (1995). What's become of research on the cultural basis of cognitive development. American Psychologist, 50, 859-877.

Seidl de Moura, M. L. (1999). Interação mãe-bebê e o desenvolvimento infantil: um estudo longitudinal e transcultural. Projeto de pesquisa não-publicado, CNPq e Universidade do Estado do Rio de Janeiro.

Seidl de Moura, M. L., Ribas, A. F. P., \& Ribas, R. C., Jr. (1998). Avaliando idéias de pais acerca da infância e do desenvolvimento. In Sociedade Brasileira de Psicologia do Desenvolvimento (Org.), Anais do II Congresso Brasileiro de Psicologia do Desenvolvimento. Gramado, RS: Autor.

Siegel, I. E., \& McGillicuddy-De Lisi, A. V. (2002). Parent beliefs are cognitions: the dynamic belief systems model. In M. H. Bornstein (Org.), Handbook of parenting, Vol. 3: Being and becoming a parent (2 $2^{\underline{a}}$ ed., pp. 485-508). Mahwah, New Jersey: Lawrence Erlbaum.

Trindade, Z. A (1993). As representações sociais e o cotidiano: a questão da maternidade e da paternidade. Psicologia: Teoria e Pesquisa, 9, 535-546.

\section{Notas}

1 Os autores agradecem aos colegas professores, e aos alunos de graduação, bolsistas de iniciação científica e mestrandos que colaboraram de diversas maneiras em cada cidade. Belém: Carlos Benedito Pereira da Paixão, Celi da Costa Silva Bahia, Lene Wilma Rodrigues Lopes, Lilia Iêda Cavalcante, Marcelo de Araújo Marques e 
Neide Maria Fernandes de Souza; Porto Alegre: Daniela Lindenmeyer, Gisele Pinheiro dos Santos, Luana Klein de Souza e Tonantzin Ribeiro; Rio de Janeiro: Flávia Ferreira da Silva Costa, Gabriela Aparecida Fructuoso de Brito, Giselle do Couto Costa e Juliana Rodrigues Ramos: Salvador: Aila Cabral, Carla Oliveira Sampaio, Cristina Goulart, Daniele Vilas Boas, Profa. Elaine Rabinovich, Hermano Trineto, Lia da Rocha Lordelo, Nayara Nascimento Rego, Patrícia Silva de Santana, Roberta Ferreira Takei e Sheila de Quadros Uzêda. Agradecem também às mães que colaboraram com a pesquisa e às entidades de fomento que apóiam a professores e alunos desse grupo: CNPq e FAPERJ. Finalmente, desejam expressar sua gratidão ao Dr. Marc Bornstein, do National Institute of Child Health and Development, do Estados Unidos, por ter disponibilizado a versão em inglês do instrumento utilizado.

2 Os dois primeiros autores organizaram e coordenaram o presente estudo; os três primeiros foram os principais responsáveis pela redação do artigo. Ana Cecília de Souza Bastos, Celina Maria Colino Magalhães, Mauro Luís Vieira e Nádia Maria Ribeiro Salomão coordenaram as equipes nos Estados e colaboraram na redação do artigo. Seus nomes são apresentados em ordem alfabética. Seguem-se os nomes das colaboradoras desse artigo cujos dados coletados em suas regiões foram também incluídos em suas respectivas dissertações de Mestrado.

3 Mestranda do Programa de Pós-graduação em Psicologia Social da Universidade Federal da Paraíba, sendo orientada pela Profa. Dra. Nádia Maria Ribeiro Salomão.

4 O trabalho realizado em Santa Catarina constituiu parte da Dissertação de Mestrado dessa autora, sob orientação do Prof. Dr. Mauro Luís Vieira.

5 O Índice de Desenvolvimento Humano foi criado originalmente para medir o nível de desenvolvimento humano dos países a partir de indicadores de educação, longevidade e renda (www.pnud.org.br). O índice varia de 0 (nenhum desenvolvimento humano) a 1 (desenvolvimento humano total). Para se aferir o IDH dos municípios de um país as dimensões são as mesmas. O IDH de 2000 apresenta os seguintes valores e posição relativa de cada município investigado no presente estudo: Porto Alegre $\left(0,86 ; 11^{0}\right.$ no país); Rio de Janeiro $\left(0,84 ; 60^{\circ}\right.$ no país); Belém $\left(0,80 ; 444^{\circ}\right.$ no país); Salvador $\left(0,805 ; 471^{\circ}\right.$ no país); Itajaí $\left(0,803 ; 507^{\circ}\right.$ no país $)$ e João Pessoa $\left(0,78 ; 985^{\circ}\right.$ no país). O IDH do Brasil é 0,75 , ocupando o $75^{\circ}$ lugar entre os países do mundo.

Maria Lucia Seidl de Moura, doutora em Psicologia Cognitiva pela Fundação Getúlio Vargas, é professora na Universidade do Estado do Rio de Janeiro. Endereço para correspondência: Rua Fritz Feigl, 465, Eldorado, Freguesia; Jacarepaguá, RJ; CEP 22750-600. Tel.: (21) 4471-588. Fax: (21) 587-7284. E-mail:mlseidl@alternex.com.br Rodolfo de Castro Ribas Jr., doutor em Psicologia Social pela Universidade do Estado do Rio de Janeiro, é professor na Universidade Federal do Rio de Janeiro.

César Augusto Piccinini, doutor em Psicologia por University College of London (Reino Unido), é professor no Instituto de Psicologia da Universidade Federal do Rio Grande do Sul.

Ana Cecília de Sousa Bastos, doutora em Psicologia pela Universidade de Brasília, é professora na Universidade Federal da Bahia.

Celina Maria Colino Magalhães, doutora em Psicologia Experimental pela Universidade de São Paulo, é professora na Universidade Federal do Pará.

Mauro Luís Vieira, doutor em Psicologia Experimental pela Universidade de São Paulo, é professor na Universidade Federal de Santa Catarina.

Nádia Maria Ribeiro Salomão, doutora em Psicologia pela University of Manchester (Reino Unido), é professora na Universidade Federal da Paraíba.

Algeless Milka Pereira Meireles da Silva é mestranda em Psicologia Social na Universidade Federal da Paraíba. Anna Karina da Silva é mestranda em Psicologia na Universidade Federal de Santa Catarina. 